\title{
5 nm Gold-Ni-NTA binds His Tags
}

\author{
V. Reddy*, E. Lymar*, M. Hu*, and J. F. Hainfeld* \\ * Biology Department, Brookhaven National Laboratory, Upton, NY 11973
}

Molecular biology has benefited by tags encoded into expressed proteins for their identification and purification. The protein of interest is constructed with DNA that includes an added "tail" sequence coding for a string of amino acids that bind to an antibody, a metal column, or a some other binding couple. Common genetic tags include 6x-histidines that bind Ni-NTA, Flag that binds to anti-flag antibodies, strep-tag that binds to strep-tactin, c-myc that binds to antibody, calmodulin binding domain that binds to calmodulin, cellulose binding domain that binds cellulose, glutathione-S-transferase that binds glutathione, streptavidin binding peptide that binds streptavidin, and a number of others.

These genetic constructs can also be used for protein localization and tracking within a cell. Although green fluorescent protein has been similarly programmed in as a "tail" fusion protein for following proteins in a cell by light microscopy, EM requires a dense label, such as gold. One may use anti-GFP gold labels, but another approach is to derivatize gold nanoparticles with the binding couple for the tags commonly used in protein expression. The tags can be much smaller that GFP, and the binding couples can be much smaller than antibodies. For example, a $1.4 \mathrm{~nm}$ gold particle synthesized with the nitrilotriacetic acid (NTA) group charged with nickel was previously shown to bind specifically to $6 \mathrm{x}$-His tagged proteins with visualization by EM [1].

Here we extend this technology to a larger $5 \mathrm{~nm}$ gold particle. The purpose for a larger particle is for better visibility directly in the TEM. The $1.4 \mathrm{~nm}$ particle is directly visible in ice or on a thin film, but becomes difficult to see in stains or in thicker samples, such as tissue sections. Although silver or gold enhancement that grows the particle is possible, a $5 \mathrm{~nm}$ particle may be seen directly without enhancement, and reduce the steps required for preparation. Enhancement also leads to more particle size variation, and this is also avoided.

Fig. 1 shows the derivatized $5 \mathrm{~nm}$ gold particles in TEM. When incubated with the protein ISWI from the ACF chromatin remodeling complex, synthesized with a His tag, a conjugate peak appeared in the chromatogram (Fig. 2), and its spectrum indicated both gold and protein presence. A control experiment was to incubate the Ni-NTA-5 nm with ISWI without the 6x-His tag. In this case, virtually no binding to the protein was seen as evidenced by the absence of the conjugate peak (Fig. 2).

\section{References}

[1] J. Hainfeld et al., J. Struct. Biol. 127 (1999)185. 


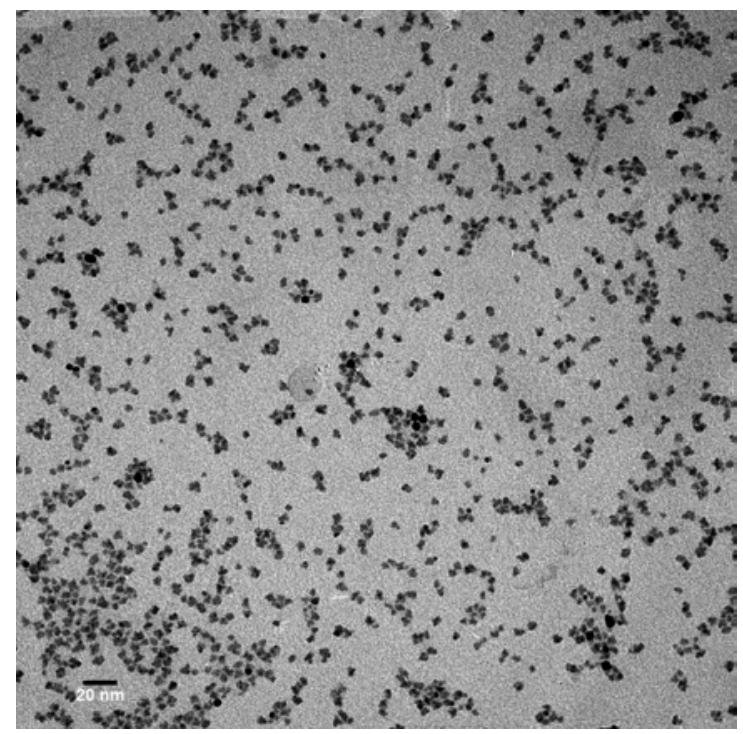

Fig. 1. TEM image of functionalized $5 \mathrm{~nm}$ gold particles.
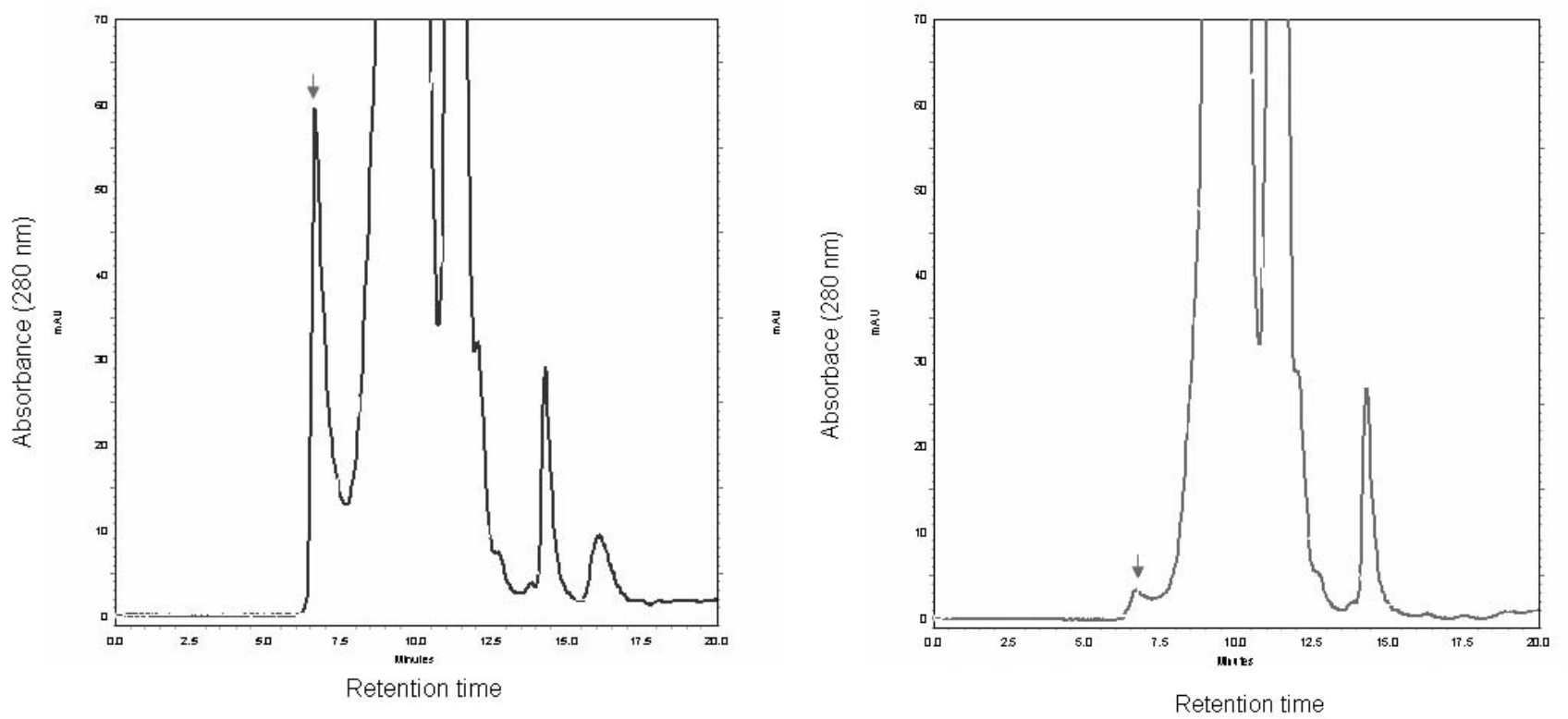

Fig. 2. Left: Chromatogram showing new peak (arrow) when 6x-His protein (ISWI) is incubated with Ni-NTA $5 \mathrm{~nm}$ gold. Right: Control experiment of incubation of 5nm-Ni-NTA with ISWI without $6 \mathrm{x}-\mathrm{His}$ tag. No significant new peak is formed (arrow). 